\title{
Revista de Psicología
}

Vol. 17 (2), 2015, pp. 137-149 (ISSN 2411-0167)

doi: 10.18050/revpsi.v17n2a7.2015

\section{ANTAGONISMOS ENTRE CONCEPCIONES DE EMPATÍA Y SU RELACIÓN CON LA CONDUCTA PROSOCIAL}

Sofía Esmeralda Auné Universidad de Buenos Aires

Facundo Juan Pablo Abal Universidad de Buenos Aires

Horacio Felix Attorresi Universidad de Buenos Aires

Cómo citar este artículo: Auné, S; Abal, F. \& Attorresi, H. (2015). Antagonismos entre conceptciones de empatía y su relación con la conducta prosocial. Revista de Psicología, 17(2), 137-149. doi: 10.18050/revpsi.v17n2a7.2015.

La correspondencia relacionada con este artículo debe dirigirse a Sofía Esmeralda Auné, e-mail: sofiaaune177@hotmail.com. Virrey Liniers 577 15º B (1220), CABA; Buenos Aires - Argentina. 


\title{
ANTAGONISMOS ENTRE CONCEPCIONES DE EMPATÍA Y SU RELACIÓN CON LA CONDUCTA PROSOCIAL
}

\author{
Auné, Sofía Esmeralda*; Abal, Facundo Juan Pablo** y Attorresi, Horacio Félix*** \\ Universidad de Buenos Aires
}

\begin{abstract}
RESUMEN
Este trabajo tiene por objetivo presentar tres antagonismos cuya discusión resulta imprescindible para el avance en la comprensión de la empatía: a) Enfoque disposicional vs. enfoque situacional, b) Perspectiva cognitiva vs. perspectiva afectiva, c) Empatía como predictor vs. empatía como dimensión de la conducta prosocial. Estas discusiones son parte de una larga serie de desacuerdos que signaron el desarrollo del concepto. La falta de consenso condujo a la proliferación de diversas aproximaciones teóricas así como también las investigaciones experimentales mostraron resultados muy diversos. Sin embargo, la tendencia actual se dirige hacia el surgimiento de teorías integradoras y multidimensionales. Es así que actualmente se considera a la empatía como una conjunto de constructos que incluye reacciones cognitivas y emocionales.

Palabras clave: conducta prosocial, empatía, antagonismos
\end{abstract}

\section{ANTAGONISMS BETWEEN CONCEPTIONS OF EMPATHY AND THEIR RELATION WITH PROSOCIAL BEHAVIOR}

\begin{abstract}
This study aims to present and discuss three antagonisms which are vital to a better understanding of empathy: a) dispositional approach vs. situational approach, b) cognitive perspective vs. affective perspective, c) empathy as predictor vs. empathy as a dimension of prosocial behavior. These discussions are part of a long series of disagreements which have marked the development of the concept. This lack of consensus has led to the proliferation of diverse attempts at a theoretical definition and thus experimental research has shown very different findings. However, the current trend is moving towards the emergence of comprehensive and multidimensional theories. This is why empathy has come to be considered a set of constructs including cognitive and emotional reactions.

Key words: prosocial behavior, empathy, antagonisms

\footnotetext{
* Lic. y Prof. en Psicología. Facultad de Psicología, Universidad de Buenos Aires, Argentina. Correspondencia: sofiaaune@psi.uba.ar

** Dr. en Psicología. Facultad de Psicología, Universidad de Buenos Aires, Argentina. Jefe de Trabajos Prácticos. Correspondencia: fabal@psi.uba.ar

*** Lic. en Ciencias Matemáticas. Facultad de Psicología, Universidad de Buenos Aires, Argentina. Correspondencia: hattorre@psi.uba.ar
} 


\section{ANTAGONISMOS ENTRE CONCEPÇÕES DE EMPATIA E SUA RELAÇÃO COM A CONDUTA PRÓ-SOCIAL}

\section{RESUMO}

Este trabalho tem o objetivo de apresentar três antagonismos cuja discussão resulta imprescindível para o avanço na compressão da empatia: a) Enfoque disposicional vs. enfoque situacional, b) Perspectiva cognitiva vs. Perspectiva afetiva, c) Empatia comopreditorvs, empatia como dimensão da conduta pró-social. Estas discussões fazem parte de uma longa série de desacordos que marcaram o desenvolvimento do conceito.A falta de consenso levou à proliferação de diversas aproximações teóricas como também mostraram resultados diferentes das pesquisas experimentais. No entanto, a tendência atual caminha em direção ao surgimento de teorias integradoras e multidimensionais. É assim que atualmente considera-se a empatia como um conjunto de construtos que inclui reações cognitivas e emocionais.

Palavras-chave: conduta pró-social- empatia - antagonismos

El concepto de empatía tiene una historia signada por el desacuerdo. Aunque ha sido estudiada por cientos de años con contribuciones de la filosofía, teología, psicología del desarrollo, social y de personalidad, etología y neurociencia, el campo continúa sufriendo la falta de consenso acerca de la naturaleza del fenómeno (Preston, \& De Waal, 2002). Las investigaciones experimentales ofrecen resultados muy diversos, dado que las concepciones de las que se parte para definirlo son disímiles e incluso, en algunos casos, antagónicas (Fernández Pinto, López Pérez, \& Márquez, 2008; Preston \& De Waal, 2002). Esta multiplicidad se transforma en una dificultad cuando surge la necesidad de investigar el concepto en forma precisa (Olivera Ryberg, 2010).

Una de las conceptualizaciones más extendidas sobre la empatía la define como un conjunto de constructos que incluyen los procesos de ubicarse en el lugar del otro, reacciones cognitivas y emocionales del observador ante una situación determinada y respuestas tanto afectivas como no afectivas que resultan de esos procesos (Davis, 1996). Según Batson y Ahmad (2009) en los últimos años el término empatía ha sido aplicado para cuatro estados psicológicos diferentes.
Dos de ellos se refieren a formas de la toma de perspectiva y los restantes a formas de respuesta emocional. Más recientemente se ha delimitado a los comportamientos empáticos como aquellas conductas que manifiestan comprensión, refuerzo y soporte emocional (Auné, Abal, \& Attorresi, 2015).

¿Qué es propiamente la empatía dentro de la esfera de la afectividad? ¿Cuáles son los elementos que la forman? ¿Cuál es su efecto real en el desarrollo de la personalidad del sujeto? ¿Es una conducta prosocial (Hay, \& Cook, 2007) o -como lejanamente sostenía Lipps (1903)- un comportamiento en el que se ignora la alteridad, considerado el otro una invención del yo, al referirse la empatía al sí mismo, considerada sólo como una vivencia que podría no incluirlo?

Esta falta de consenso ha conducido a la proliferación de aproximaciones teóricas dirigidas a determinar si la empatía es un proceso emocional o cognitivo y a distinguir la empatía del contagio emocional, la simpatía y la toma de perspectiva. En un nivel profundo, el debate parece surgir de la falta de un mecanismo explicativo (Preston, \& De Waal, 2002). Citando a Allport (1968, en Wispé, 1987) “el 
proceso de la empatía recuerda a un acertijo en psicología social”.(p. 30)

En la actualidad se hallan en la literatura, como ya se mencionó, posturas antagónicas acerca de la empatía. Tras una revisión, se han seleccionado tres frentes de disputa o antagonismos cuya discusión resulta imprescindible para el avance en la comprensión del constructo:

1) Enfoque disposicional vs. enfoque situacional

2) Perspectiva cognitiva vs. perspectiva afectiva

3) Empatía como predictor vs. empatía como dimensión de la conducta prosocial

Se plantea como objetivo de este trabajo revisar dichos antagonismos y analizar la existencia de enfoques integradores de los mismos.

Desarrollo

\section{Orígenes del concepto de empatía}

Según mencionan Royzman y Rozin (2006), el filósofo Adam Smith fue principal referente de la doctrina del liberalismo económico, quien realizó una de las primeras alusiones acerca de la empatía. Escribe este autor en su libro La Teoría de los sentimientos morales (1759) que otro pensador británico, Thomas Hobbes, sostenía que el hombre, un ser en esencia egoísta, se ve impulsado a refugiarse en la sociedad, no por ningún amor natural hacia sus semejantes sino porque, faltándole la colaboración de los otros, es incapaz de subsistir ante los peligros. Contrariando a Hobbes, Smith entendía que esa actitud egocéntrica del ser humano no elimina la idea de que "existen evidentemente en su naturaleza algunos principios que le hacen interesarse por la suerte de otros, y hacen que la felicidad de estos le resulte necesaria, aunque no derive de ella nada más que el placer de contemplarla" (Smith, 1759/1997, p. 49). A este sentimiento lo describió en sus dos manifestaciones: la simpatía o solidaridad frente al dolor y el ejercicio de la solidaridad con alegría (Smith, 1759/1997).

Otros importantes precursores del concepto de empatía fueron David Hume y Arthur Schopenhauer. Hume destacó la existencia de una propensión a percibir comunicaciones emocionales aunque sean diferentes del propio sentir (Wispé, 1991). Se convierte así la idea de la emoción de otro en la emoción en sí, que puede ser tan fuerte como la original. Schopenhauer destacó que cuando otra persona sufre el observador sabe que ese sentimiento es ajeno, pero al mismo tiempo lo percibe casi como que fuera propio (Wispé, 1991).

Siguiendo a Berthoz y Jorland (2004) fue Vischer quien en 1873 introdujo el término Einfühlung en el sentido de "transferencia inconsciente (Versetzen) de la propia forma corpórea, y a su vez también del alma, a la forma del objeto" (Vischer, 1873/2003, p. 42). Lo hizo para explicar las respuestas generadas por la observación de pinturas. Describió cómo formas particulares generan reacciones emocionales específicas. La Einfühlung es una vivificación de la imaginación; junto a ella se manifiestan la Anfühlung (animación de la sensibilidad), la Zufühlung (recreación de la forma) y la Nachfühlung (sentido de la forma expresada simbólicamente). Lipps fue el primero en sugerir una explicación para la Einfühlung (1903). La conceptualizó primeramente como una tendencia de los observadores a proyectarse en los objetos percibidos y la aplicó posteriormente al entendimiento de la persona. Hipotetizó que se comprenden los sentimientos de otros mediante un proceso de inferencia analógica basado en la imitación de las expresiones ajenas de forma imaginaria (Wispé, 1991). Según Lipps, la percepción de un gesto emocional en otra persona activa directamente la misma emoción en el 
perceptor, sin ningún proceso de asociación o de toma de perspectiva cognitiva interviniente. El término Einfühlung se tradujo al inglés por Titchener como Empátheia, que originalmente significaba pasión y que se compone del prefijo en (dentro) y páthos (sentimiento), es decir un sentimiento internalizado (Davis, 1996).

En el marco de la teoría psicoanalítica se concibe que la empatía entre dos seres surge a partir de la identificación (Freud, 1921/1992). Para este autor hay un camino que lleva desde la identificación, por la imitación, a la empatía. Es decir, a la comprensión del mecanismo por el cual el ser humano es capaz de interesarse y acortar la distancia hacia cualquier actitud de la vida de otro ser. El niño, desde la identificación, reduce su ansiedad. Desde una perspectiva psicoanalítica se asume que, generalmente, la ansiedad es generada internamente por la excitación de los impulsos reprimidos que son desplazados y proyectados sobre acontecimientos ambientales. En el adulto, el afán de identificación puede perdurar también como un mecanismo de defensa contra la ansiedad. Tanto en el niño como en el adulto, la identificación puede expresar emociones de sumisión amorosa o de agresión, es decir, que es un proceso ambivalente.

Freud (1921/1992) entiende que la identificación es la forma más originaria de ligazón afectiva con un objeto. En el niño, suele sustituir una ligazón libidinosa de objeto por la vía regresiva mediante la introyección del objeto en el propio yo. No es que se producen pulsiones sexuales sino que los bebés, al percibir algún desvalimiento, tienen una imperiosa necesidad de alcanzar total comunidad con quien le cuida, habitualmente su madre. Este proceso infantil se repite en la vida adulta. La identificación ha de ser más exitosa cuando el individuo encuentra una significativa comunión de intereses con otro y, así, se produce el comienzo de una nueva ligazón.

Otra definición del concepto de empatía bajo la denominación endopatía. Este término designa a la participación afectiva y emotiva de un sujeto en una realidad que le es ajena (Ferrater Mora, 2000). La participación en cuestión puede ser a su vez consciente o inconsciente. Los autores que admiten la posibilidad de semejante participación afectiva suelen destacar que solo mediante ella puede alcanzarse una muy profunda comprensión de ciertos fenómenos o procesos externos a la subjetividad (Merleau Ponty, 1948/2003). Se entiende así por empatía, desde una perspectiva fenomenológica, a la participación afectiva en realidades anímicas o de algún modo relacionadas con lo anímico y con el otro, el distinto de uno. Una prosecución del pensamiento del otro a través de la palabra, una reflexión en el otro, "un poder de pensar según el otro que enriquece nuestros propios pensamientos" (Merleau Ponty, 1948/2003; p. 196).

En muchos casos, la empatía se refiere a una sintonía con un estado emocional negativo pero también es posible para estados positivos (Wispé, 1978). Justamente, Merleau Ponty (1948/2003) considera que es el cuerpo aquello que le asegura al individuo que existen las personas y los objetos. Por lo tanto, el cuerpo no es un ente cualquiera del mundo: es un medio de comunicación entre el sujeto y el mundo. Si existe la posibilidad de ver, es gracias a que el sujeto tiene un cuerpo y, dado este fenómeno, puede percibir a los otros, a los objetos. El cuerpo es el horizonte de la percepción. Para Merleau Ponty, un otro se entrega, como no puede ser de otro modo, a 
través de su cuerpo: "Los otros hombres jamás son para mí puro espíritu: sólo los conozco a través de sus miradas, sus gestos, sus palabras, en resumen, a través de su cuerpo" (Merleau Ponty, 1948/2003, p. 48).

La emoción -sea cual fueremodificaría el estado somático produciendo signos corporales que son los que se ofrecen a la mirada de los demás para que al otro le resulte posible aprehender las emociones del que las expresa. Suscita en él una reacción empática situando a ambos cuerpos en una relación que les impone respuestas similares en una dinámica de diálogo que, al influir afectivamente al Yo de ambos interlocutores, les provoca emociones iguales y permite a cada uno vivenciar tanto la predisposición espiritual como el cuerpo del otro (Merleau Ponty, 1948/2003).

\section{Empatía disposicional vs. Empatía situacional}

La teoría de empatía disposicional se contrapone a la teoría de la empatía situacional. Para la primera existen personas con diferente grado de empatía de forma independiente de la situación; es decir, entiende a la empatía como una tendencia relativamente estable de la persona a percibir y experimentar las emociones de otros. Mientras que para la segunda existen aspectos situacionales, contextos físicos o relacionales concretos que generan más o menos empatía (Otiz, Apodaka, Etxeberría, Ezeiza, Fuentes, \& López, 1993; Sánchez Queija, Oliva, \& Parra, 2006).

Se han utilizado cuestionarios (tanto autoinformes como informes de otros) para evaluar las tendencias empáticas disposicionales. En cambio, para evaluar la empatía situacional se emplean generalmente los estímulos inducidos por las respuestas faciales, verbales o autonómicas así como las manipulaciones de conjuntos de observación.

Mehrabian y Epstein (1972), referentes de la teoría disposicional, consideran a la empatía como una respuesta emocional vicaria que se experimenta ante las emociones ajenas y que permite sentir lo que otro individuo siente. Encontraron una relación significativa entre la tendencia empática y el comportamiento de ayuda que, sostienen, sustenta la idea de que las personas empáticas son más receptivas emocionalmente a las necesidades ajenas. Además, las personas con mayor tendencia empática resultan ser más sensibles a las experiencias emocionales de otros, tanto de cualidad positiva como negativa.

Para Batson y Shaw (1991), representantes de la teoría de la empatía situacional, la fortaleza del vínculo con la persona en necesidad se relaciona directamente con la posibilidad de adoptar su perspectiva. Adoptar la perspectiva de la persona en necesidad parece ser una precondición de la emoción empática. Si esto ocurre, la magnitud de dicha emoción es una función de dos factores: a) magnitud de la necesidad percibida y b) fuerza del vínculo. La empatía se concibe como una emoción que se suscita ante la presencia de estímulos situacionales concretos e implica competencias específicas.

Actualmente la teoría disposicional es la que ha ganado más terreno ya que en la mayoría de los estudios se considera a la empatía como un factor diferenciador entre individuos donde algunos la tienen en mayor medida que otros (e.g. Allen, 2003; Farrington, \& Jolliffe, 2001), es decir, como parte de la personalidad. Ha habido también una proliferación de teorías integradoras entre lo disposicional y lo situacional (e.g. Salovey, \& Mayer, 1990). 


\section{Perspectiva cognitiva vs. Perspectiva afectiva de la empatía}

Aunque primariamente la empatía fue definida en términos afectivos que intentaban explicar el mecanismo por el cual se compartían las emociones (e.g. (Lipps, 1903), rápidamente el énfasis cambió hacia una perspectiva cognitiva. Esta variación se debió a la influencia de los trabajos de Mead (1934) y Piaget (1932). Desde entonces, se han realizado múltiples estudios desde un enfoque cognitivo de la empatía. En la década de los 50, se destacan los trabajos de Chapin (1942), Dymond (1950) y Kerr y Speroff (1954). Hogan (1969) definió la empatía como la metarrepresentación de los estados mentales ajenos. Estos estudios consideran a la empatía como toma de perspectiva acerca de los sentimientos y pensamientos de otros. Actualmente, se ha considerado que la empatía como capacidad cognitiva es similar al concepto de Teoría de la Mente (e.g. Baron Cohen et al., 2004).

La Teoría de la Mente es definida como habilidad de explicar y predecir el comportamiento propio y ajeno mediante la atribución de estados mentales independientes (Gallagher, \& Frith, 2003). Los trastornos en la capacidad empática y el autismo serían consecuencias directas de fallas en esta habilidad la cual estaría asentada en mecanismos biológicos específicos (Elliott et al. 2006). Se observa así como un desorden en principio cognitivo conduce a consecuencias sociales (Baron Cohen et al., 1997).

Pese a estos aciertos de las teorías cognitivas, desde la década de los '60, se empezó a consolidar una visión distinta de la empatía, que concedía más importancia a su componente afectivo que al cognitivo, definiéndola como un afecto compartido o bien como un sentimiento vicario (Stotland, 1969). Esta noción de empatía surge de los estudios realizados por diversos investigadores acerca de la compasión y el altruismo. Dentro del enfoque afectivo, las definiciones de empatía varían en cuanto a la amplitud o la restricción con la que diferentes sujetos manifiestan su emoción en respuesta a la emoción del otro.

Baron-Cohen y Wheelwright (2004) afirman que existen cuatro variedades de empatía, aunque cuestionan severamente la que ellos mismos incluyen como tres que adjudican a Stotland (1969). Son las siguientes:

1) La sensación en el observador provocada por la sensación de la persona observada (por ejemplo: "sientes miedo cuando ves a alguien con mayor temor");

2) Una sensación en el observador que procede simplemente del estado emocional de la otra persona (por ejemplo, sentir piedad por otro que está evidentemente muy triste);

3) La sensación en el observador de que puede emitir una respuesta emocional extraña o paradójica a la emoción del otro (por ejemplo, sentir placer ante el dolor ajeno). Baron Cohen y Wheelwright (2004) no consideran que ese estado pueda denominarse empático.

4) La sensación en el observador de preocupación o de compasión ante una señal o pedido de socorro.

Actualmente Baron Cohen y Wheelwright (2004) señalan que son dos los componentes de la empatía: el afectivo y el cognitivo. Lo grafican con la siguiente imagen: 


\section{- FIGURA 1. Componentes de} la empatía.

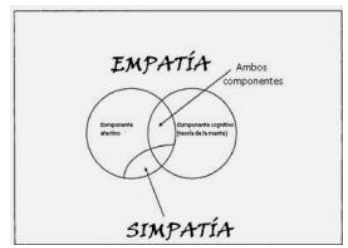

Figura 1. El modelo muestra dos componentes de la empatía que, a su vez, se pueden solapar y cómo la empatía es un caso especial del componente afectivo de la empatía. Baron Cohen y Wheelwright (2004)

Baron Cohen y Wheelwright (2004) realizaron dos estudios sobre el cociente de empatía (EQ) en adultos de inteligencia normal mediante un test de 40 ítems puntuado entre 0 y 80 . En uno de los estudios realizados entrevistaron a 197 individuos (con prevalencia de mujeres: 126) en el cual confirmaron una capacidad femenina para la endopatía significativamente más alta que la de los hombres. Las mujeres obtuvieron en promedio un puntaje de 47.2 y los hombres un puntaje de 41.8 . El dato estadístico más interesante reveló que fueron tres mujeres por cada hombre las que anotaron el puntaje máximo alcanzado.

En esta línea se tienen en cuenta ambas visiones de la empatía (cognitiva y afectiva) desde una perspectiva situacional. Para Salovey y Mayer (1990), la reacción empática es posible tanto por habilidades emocionales como por competencias cognitivas. De hecho, entonces, no puede dejarse de lado el aspecto cognitivo de la empatía por cuanto es el que permite, una vez interiorizadas las percepciones $\mathrm{y}$ acciones, que la persona las transforme y pueda operar con ellas -según su capacidad y competencia- de manera reversible.

Este proceso de transformación es lo que posibilita al sujeto poner en práctica, por ejemplo, su competencia de abstracción. Por un lado analizar la situación: es decir, separa, segmenta, divide en fracciones, para poder encontrar en cada parte el núcleo principal de un suceso. Posteriormente, ejercitará una síntesis que es la conclusión de su proceso mental u operación intelectual. Cuando se habla de abstracción se habla también de atención, que es una compleja función cerebral: la atención es un estado neurocognitivo cerebral de preparación que precede a la abstracción, a la percepción, a las emociones y a la acción: "La atención focaliza selectivamente nuestra consciencia para filtrar el constante fluir de la información sensorial, resolver la competencia entre los estímulos para su procesamiento en paralelo y reclutar y activar las zonas cerebrales para temporizar las respuestas apropiadas" (Estévez González et al., 1997, p. 1989).

Primeramente fue Feshbach (1987) quien destacó la naturaleza multidimensional de la empatía. Los componentes cognitivos y emocionales coexisten en su modelo, pero las habilidades cognitivas son consideradas prerrequisitos para la empatía. En otras palabras, para Feshbach ser capaz de reconocer las emociones de otro individuo y su punto de vista es necesario pero no suficiente para empatizar con sus sentimientos.

Posteriormente, otros autores han propuesto abordajes multidimensionales para el estudio de la empatía (e.g., Davis, 1996; Hoffman, 2001; Strayer \& Roberts, 1997).

Davis (1996) aproxima una definición multidimensional de la empatía y un nuevo instrumento para su evaluación. Su Interpersonal Reactivity Index (IRI) es una importante medida de autoinforme para la evaluación de la empatía. El sujeto debe responder en qué grado lo describen distintas afirmaciones acerca cómo siente $\mathrm{y}$ 
piensa en distintas situaciones (Mestre Escrivá et al., 2004).

Aunque existen diferencias entre sus modelos, estos autores acuerdan en que los diferentes componentes de la empatía pueden ser considerados y medidos de forma separada, pero que sólo desde una visión conjunta se puede entender un constructo tan complejo (Hoffman, 2001).

\section{Empatía como predictor vs. empatía como dimensión del comportamiento prosocial}

Se ha resaltado la relevancia de la empatía en la disposición prosocial de las personas y su función inhibidora de la agresividad (Moya Albiol, Herrero, \& Bernal, 2010). Sin embargo, aunque la relación entre empatía y prosocialidad viene siendo estudiada desde hace años prevalece aún una importante indefinición conceptual e incluso de resultados (Auné, Blum, Abal, Lozzia, \& Attorresi, 2014; Sánchez-Queija, Oliva, \& Parra, 2006).

MestreEscrivá et al. (2004) registraron que las subescalas de empatía del Interpersonal Reactivity Index (IRI), en términos generales, correlacionan positivamente con la conducta prosocial y con los estilos de razonamiento prosocial más orientados a una disposición prosocial, mientras que la correlación es negativa con la conducta agresiva y con la inestabilidad emocional. Las correlaciones más fuertes se alcanzaron entre los factores de empatía que indican una disposición empática más madura y la conducta prosocial y entre estos mismos factores $\mathrm{y}$ el razonamiento internalizado. Este razonamiento incluyó argumentos orientados a la comprensión del problema del otro, a anticipar consecuencias físicas y emocionales que se puedan derivar de la acción de ayudar o no ayudar y argumentos como la satisfacción personal de actuar según los propios valores. Por el contrario, las correlaciones son negativas con el razonamiento hedonista, centrado en el beneficio personal que se puede derivar de la acción y con el razonamiento orientado a buscar la aprobación de los demás propio de una menor madurez prosocial (Mestre Escrivá et al., 2004).

Mestre Escrivá et al. (2002) plantean a la empatía como factor modulador de la conducta prosocial. Funciona como un mecanismo regulador, favoreciendo la conducta prosocial e inhibiendo la agresividad. Según los resultados que obtuvieron, la empatía aparece como el principal motivador de la conducta prosocial en sus componentes cognitivos (la comprensión del otro), pero más fuertemente en sus componentes emocionales (la preocupación por el otro). En este sentido, el actuar prosocialmente supone la utilización de criterios de igualdad y bien social y un razonamiento centrado en una orientación empática abierta (Mestre Escrivá et al., 2006).

De esta forma, la empatía sería un prerrequisito para la conducta prosocial pero no parte de la misma. El compartir los sentimientos de otra persona no necesariamente implica que se actuará, o incluso que se sentirá impelido a actuar de forma comprensiva o simpática (Mestre Escrivá et al., 2002).

Caprara et al. (2005) introducen un importante giro conceptual al incorporar a la empatía como una dimensión de la prosocialidad. Destacan la novedad de esta inclusión y afirman que la misma se basó en el convencimiento de que en la adolescencia y adultez los motivos o predisposiciones empáticas no son sólo un correlato de la tendencia a actuar prosocialmente sino que forman parte de dicha tendencia. La empatía es un componente integral de la prosocialidad en estas etapas ya que el acudir al rescate de los demás implica la capacidad de sentir sus necesidades y 
estados de ánimo (Eisenberg, \& Fabes, 1998; Hoffman, 2000).

Por lo tanto, Caprara et al. (2005) sostienen la hipótesis de que comportamientos diferentes tales como ayudar, confortar y empatizar son expresiones de la misma tendencia o disposición de orden superior denominada prosocialidad (Eisenberg \& Fabes, 1998; Hoffman, 2000). En particular, la prosocialidad en la adolescencia y la edad adulta va más allá de ayudar, cuidar y compartir, el comportamiento también se puede poner en práctica por simple educación, convención o conveniencia e implica una particular inclinación a participar en las experiencias emocionales de los demás. Básicamente, se trata de la capacidad de implicación emocional combinada con plena conciencia de las intenciones y los efectos de las acciones (Caprara et al., 2005).

La discusión entre la empatía como predictor y la empatía como parte de la tendencia prosocial aún no ha sido saldada. Ambas partes encuentran evidencia para su posición, en el primer caso mediante la obtención de correlaciones significativas con criterios externos y en el segundo, hallando que, aún incluyendo a la empatía, en un instrumento para la medición de la prosocialidad en adultos resulta confirmada una estructura interna unidimensional (Caprara et al., 2005).

\section{Comentarios}

La disputa acerca de la empatía disposicional o situacional ha sido enriquecida con el concepto de interacción entre estos dos factores. El campo de la personalidades frecuentemente considerado como la organización y estructuración de todas las dimensiones evaluables de las diferencias individuales, a través del tiempo y las situaciones (Saklofske, \& Zeidner, 1995) siendo la empatía parte de dicho campo de estudio.
Uno de los temas más polémicos en la investigación de la empatía es si resulta mejor conceptualizarla como una construcción afectiva, una construcción cognitiva, o ambas. Mientras que la investigación contemporánea parece haber adoptado la opción ambas, no se apoya de forma generalizada y el debate continúa sobre la definición y medición de la empatía. Ambas entidades (emoción y cognición) se combinan para generar empatía y establecer las principales influencias en las conductas de acercamiento y aceptación de personas o de objetos (Newman et al., 2010). Se puede afirmar, por lo tanto, que la empatía es no sólo efecto de una respuesta cognitiva sino también una respuesta emocional de un observador al estado afectivo de otro sujeto.

Numerosos investigadores han intentado buscar evidencias para sostener la relación entre empatía y conducta prosocial, sobre la base de diversas teorías de la personalidad, utilizando técnicas correlacionales (e.g. Mestre Escrivá et al., 2002). Hallándose una correlación, se ha interpretado a la empatía como moduladora de la prosocialidad. Sin embargo, la correlación no implica una relación causa-efecto, la causa o las causas que operan sobre una variable determinada pueden ser otras; siendo las variables de interés bidireccionales, es decir, tanto dependientes como independientes, $\mathrm{o}$ bien ser tanto una como la otra, variables moderadoras. Por eso es valioso el aporte de Caprara et al. (2005), en tanto pone en discusión el rol de la empatía. Futuras investigaciones podrían emplear diseños dinámicos longitudinales y el modelo de ecuaciones estructurales complejas, $\mathrm{u}$ otras técnicas actuales, para dilucidar la relación entre conducta prosocial y empatía. Es que ambos constructos se han relacionado siempre desde el punto de vista conceptual, sin embargo desde el empírico las investigaciones no han 
sido concluyentes acerca de la naturaleza de esa relación.

Es necesario destacar que estos frentes; enfoque disposicional vs. enfoque situacional, perspectiva cognitiva vs. perspectiva afectiva y empatía como predictor vs. empatía como dimensión de la conducta prosocial; son parte de una larga serie de desacuerdos: capacidad empática como continuo vs. visión dicotómica, beneficiosa siempre para el otro vs. neutral, innata vs. adquirida, entre otros.

La tendencia actual se dirige hacia el surgimiento de teorías integradoras, dejando atrás los antagonismos en torno al concepto de empatía. Se destaca la necesidad de creación de enfoques multidimensionales que incluyan lo cognitivo, emocional, disposicional $\mathrm{y}$ situacional, en la búsqueda por encontrar descripciones de mayor complejidad. De esta forma, los antagonismos representan esfuerzos para sistematizar los conocimientos que van siendo superados. Alcanzar la multidimensionalidad permitirá comprender y definir la naturaleza de la empatía en términos teóricos; pero también concebir, diseñar y construir instrumentos para su medición y formas de abordaje práctico.

Parece ser cierto que, aunque la mayoría de los seres humanos no son en extremo prosociales ni antisociales, anhelan de los demás una conducta empática. En cualquier cultura, la misantropía siempre es una excepción, nunca la norma (Rifkin, 2010). Actualmente, la expresión empática y su comprensión es considerada necesaria para un desarrollo saludable del ser humano (Rifkin, 2010). Prueba de ello son las extensas investigaciones sobre el autismo, afección donde justamente habría un déficit en estas capacidades.

Referencias

Allen, T. D. (2003). Mentoring others: A dispositional and motivational approach. Journal of Vocational Behavior, 62(1), 134-154.

Auné, S., Abal, F., \& Attorresi, H. (2015). Diseño y construcción de una escala de conducta prosocial para adultos. En prensa. Revista Iberoamericana de Diagnóstico y Evaluación Psicológica.

Auné, S., Blum, G. D., Abal, F., Lozzia, G., \& Attorresi, H. (2014). La Conducta Prosocial: estado actual de la investigación. Perspectivas en Psicología, 11(2), 21-33.

Baron Cohen, S. Jolliffe, T., Mortimore, C., \& Robertson, M. (1997). Another advanced test of Theory ofmind: Evidence from very High functioning adults with autism or Asperger Syndrome. Journal of Child Psychology and Psychiatry and Allied Disciplines, 38(7), 813-822.

Baron Cohen, S., \& Wheelwright, S. (2004). The empathy quotient: an investigation of adults with Asperger syndrome or high functioning autism, and normal sex differences. Journal of Autism and Developmental Disorders, 34(2), 163-175.

Batson, C. D., \& Ahmad, N. Y. (2009). Using empathy to improve intergroup attitudes and relations. Social Issues and Policy Review, 3(1), 141-177.

Batson, C. D., \& Shaw, L. L. (1991). Evidence for altruism: Toward a pluralism of prosocial motives. Psychological Inquiry, 2(2), 107-122.

Berthoz , A., \& Jorland, G. (2004). L'Empathie., Paris, France. Editions Odile Jacob

Caprara, G. V., Capanna, C., Steca, P., \& Paciello, M. (2005). Misura e determinanti personali della prosocialità. Un approcciosociale cognitivo. 
Giornale Italiano di Psicologia, 32(2), 287-308.

Chapin, F. S. (1942). Preliminary standardization of a social insightscale. American Sociological Review, 7, 214225.

Davis, M. H. (1996). A Social Psychological Approach. Westview Press.

Dymond, R. F. (1950). Personality and empathy. Journal of Consulting Psychology, 14, 343-350.

Eisenberg N., \& Fabes R.A. (1998). Prosocial development. In N. Eisenberg (ed.), Social, emotional, and personality. In W. Damon (ed.), Handbook of child psychology. (pp. 701-778). New York: Wiley.

Elliott, R., Völlm, B., Drury, A., McKie, S., Richardson, P., \& Deakin, J. F. W. (2006). Cooperation with another player in a financially rewarded guessing game activates regions implicated in theory of mind. Social Neuroscience, Special Issue: Theory of Mind, 1(3-4), 385-395.

Estévez González, A., García Sánchez, C., \& Junqué, C. (1997). La atención: una compleja función cerebral. Revista de Neurología, 25(148), 1989-1997.

Farrington, D. P., \& Jolliffe, D. (2001). Personality and crime. In N. J. Smelser, \& P. B. Baltes (Eds.), International encyclopedia of the social and behavioural sciences (pp. 11260 - 11264). Amsterdam: Elsevier.

Fernández Pinto, I. López Pérez, B., \& Márquez, M. (2008). Empatía: Medidas, teorías y aplicaciones en revisión. Anales de Psicología, 24(2), 284-298.

Ferrater Mora, J. (2000). Diccionario de filosofía abreviado., Buenos
Aires, Argentina. Editorial Sudamericana

Feshbach, N. D. (1987). Parental empathy and child adjustment/maladjustment. In N. Eisenberg and J. Strayer (Eds.), Empathy and its development (pp. 271-291). New York: Cambridge University Press.

Freud, S. (1992). Psicología de las masas y análisis del yo. Obras completas, Tomo XVIII, Amorrortu, Buenos Aires. (Trabajo original publicado en 1921).

Gallagher, H. L., \& Frith, C. D. (2003). Functional imaging of "theory of mind". Trends in Cognitive Sciences, 7(2), 77-83.

Hay, D. F., \& Cook, K. V. (2007). The transformation of prosocial behavior from infancy to childhood. In: C. A. Brownell \& C. B. Kopp (Eds.), Socioemotional development in the toddler years: Transitions and transformations (pp. 100131)., New York, USA, The Guilford Press.

Hoffman M. L. (2000).Empathy and moral development: Implications for caring and justice. New York: Cambridge University Press.

Hoffman M. L. (2001). Toward a comprehensive empathy-based theory of prosocial moral development. In: Bohart A, Stipek D, (eds). Constructive \& Destructive Behavior: Implications for Family, School, \& Society (pp 61-86). Washington, DC: American Psychological Association.

Hogan, R. (1969). Development of an Empathy Scale. Counsulting and Clinical Psychology, 33, 307316.

Kerr, W. A., \& Speroff, B. G. (1954). Validation and evaluation of the 
empathy test. Journal of General Psychology, 50,369-376.

Lipps, T. (1903). Einfühlung, innere Nachahmung und Organ empfindung. Archivfur die Gesamte Psychologie 1, 465519.

Mead, G. H. (1934). Mind, self, and society. Chicago: University of Chicago Press.

Mehrabian, A., \& Epstein, N. (1972). A measure of emotional empathy. Journal of personality, 40(4), 525-543.

Merleau Ponty, M. (2003). El mundo de la percepción. Siete conferencias. Fondo de Cultura Económica. Argentina, Buenos Aires.

Mestre Escrivá, M. V., Frías Navarro, M. D., \& Samper García, P. (2004). La medida de la empatía: análisis del Interpersonal Reactivity Index. Psicothema, 16(2), 255-260.

Mestre Escrivá, M. V., Samper García, P., \& Frías Navarro, M. D. (2002). Procesos cognitivos y emocionales predictores de la conducta prosocial y agresiva: La empatía como factor modulador. Psicothema, 14(2), 227-232.

Mestre Escrivá, M. V., Samper García, P., Tur, A. M., Cortés, M. T., \& Nácher, M. J. (2006). Conducta prosocial y procesos psicológicos implicados: un estudio longitudinal en la adolescencia. Revista Mexicana de Psicología, 23(2), 203-215.

Moya Albiol, L., Herrero, N., \& Bernal, M. C. (2010). Bases neuronales de la empatía. Rev Neurol, 50(2), 89-100.

Newman, A., Dennis, C., Wright, L. T., \& King, T. (2010). Shoppers' experiences of digital signage-a cross-national qualitative study. JDCTA, 4(7), 50-57.
Olivera Ryberg, J. M. (2010). Acerca del concepto de empatía. Su rol $y$ evaluación en psicoterapia. Tesis para obtener el título de Licenciado en Psicología, Universidad de Belgrano, Argentina.

Otiz, M. J., Apodaka, P., Etxeberría, I., Ezeiza, A., Fuentes, M. J., \& López, F. (1993). Algunos predictores de la conducta prosocial-altruista en la infancia: empatía, toma de perspectiva, apego, modelos parentales, disciplina familiar e imagen del ser humano. Revista de Psicología Social, 8(1), 83-98.

Piaget, J. (1932). The moral judgment of the child. London: Kegan Paul, Trench, Trubner.

Preston, S. D., \& De Waal, F. (2002). Empathy: Its ultimate and proximate bases. Behavioral and brain sciences, 25(1), 1-20.

Rifkin, J. (2010). La civilización empática. La carrera hacia una conciencia global en un mundo en crisis. Barcelona. Ed. Paidós.

Royzman, E. B., \& Rozin, P. (2006). Limits of Symhedonia: The Differential Role of Prior Emotional Attachment in Sympathy and Sympathetic Joy. Emotion, 6(1), 82-93.

Saklofske, D. H., \& Zeidner, M. (1995). International handbook of personality and intelligence. Springer Science \& Business Media.

Salovey, P. \& Mayer, J.D. (1990). Emotional intelligence. Imagination, Cognition and Personality, 9, 185-211.

Sánchez Queija, I., Oliva, A., \& Parra, Á. (2006). Empatía y conducta prosocial durante la adolescencia. Revista de Psicología Social, 21(3), 259271. 
Smith, A. (1997). Teoría de los sentimientos morales. Madrid. Ed. Alianza, (Trabajo original publicado en 1759).

Stotland, E. (1969). "Exploratory investigations of empathy", in Berkowitz L. (Ed.), Advances in experimental social psychology, Vol. 4, pp. 274- 314, New York: Academic Press.

Strayer, J., \& Roberts, W. (1997). Facial and verbal measures of children's emotions and empathy. International Journal of Behavioral Development, 20(4),627-649

Vischer, R. (2003). "Sul sentimento ottico della forma", en Friedrich
T. Vischer, R. Vischer, Simbolo e forma, Aragno, trad. it. a c. di A. Pinotti, Torino, Italia.

Wispé, L. (1978). The distinction between sympathy and empathy: To call forth a concept, a word is need. Journal of Personality and Social Psychology, 50(2), 314321.

Wispé, L. (1987). History of the concept of empathy. N. Eisenberg \& J. Strayer. In: Empathy and its development. Ed. Cambridge University Press.

Wispé, L. (1991). A Summary of the Theory of Sympathy.In The Psychology of Sympathy (pp. 157-163).Springer US.

Recibido: 28 de febrero del 2015

Aceptado: 12 de agosto del 2015 